\title{
El grupo de Bogotá y la filosofía latinoamericana en la Universidad Santo Tomás: un testimonio*
}

The Bogota group and the Latin American philosophy at the University of Santo Tomás: a testimony

0 Grupo de Bogotá e a filosofia Latinoamericana na Universidade Santo Tomás: uma testemunha

Fecha de entrega: 2 de febrero de 2016

Fecha de evaluación: 5 de julio de 2016

Fecha de aprobación 30 de julio de 2016

Tere Houghton Pérez ${ }^{* *}$

\section{Resumen}

El texto aborda, a manera de testimonio, el tema de la historia del Grupo de Bogotá -surgido en la Universidad Santo Tomás- y la Filosofía latinoamericana, en las décadas de 1970 y 1980, mediante el comentario de autores y textos que han hecho el análisis y la crítica del mismo grupo. Para hacerlo, se vale de las lecciones de verano de 1988, de Peter

* Este texto hace parte de las investigaciones sobre la Filosofía Latinoamericana que la autora ha adelantado durante su vida academica. Una versión parcial de esta investiación fue presentada como lección inaugural en el XVI Congreso Internacional de Filosofia Latinoamericana, junio 30 de 2015. . DOI: http://dx.doi.org/10.15332/s0120-8462.2016.0115.03

* Profesora de la maestría en Filosofía Latinoamericana de la Universidad Santo Tomás de Bogotá. 
Sloterdijk, extraídas de su texto Venir al mundo venir al lenguaje, que aborda el tema del nacimiento como una "poética del comenzar".

Se describen cinco momentos de la vida del grupo situada en el movimiento de la filosofía liberadora en el continente y el país, así el primero, el nacimiento como desligamiento de una filosofía inocua y repetitiva; segundo, el abordaje de las necesidades o urgencias del proyecto naciente, tercero, el surgimiento creativo de las iniciativas que dieron lugar a las instituciones y mediaciones aún hoy presentes en la vida de la universidad y del país; el cuarto momento de la demora o de la pausa que se relaciona con la autocrítica; el quinto momento del trabajo de consolidación cercano a la madurez. A través del texto aparece el papel decisivo jugado en la formación del grupo por los maestros de la Filosofía de la Liberación, especialmente el maestro Enrique Dussel.

Palabras clave: Filosofía de la liberación, Grupo de Bogotá, a priori del nacimiento, poética del venir al mundo, congreso.

\section{Abstract}

IThe paper approaches, as a testimony, the theme of the history of the Bogota Group - which emerged at the University of Santo Tomás and the Latin American Philosophy in the 1970s and 1980s, through the comments of authors and texts that have made the analysis and criticism of the same group. To do so, it uses the summer lessons of 1988, by Peter Sloterdijk, extracted from his text Coming into the world coming into language, which addresses the issue of birth as a "poetics of the beginning".

Five moments of the life of the group located in the philosophy of liberation movement in the continent and the country are described, so the first, the birth as a disconnection of a harmless and repetitive philosophy; second, the approach to the needs or urgencies of the nascent project; third, the creative emergence of the initiatives that gave rise to institutions and mediations still present in the life of the university and the country; the fourth moment, the delay or pause that is related to self-criticism; the fifth moment, the consolidation work 
close to maturity. Through the text appears the decisive role played in the formation of the group by the teachers of the Philosophy of Liberation, especially professor Enrique Dussel.

Keywords: Philosophy of liberation, Bogota Group, a priori of birth, poetics of coming into the world, congress.

\section{Resumo}

O texto aborda, a maneira de testemunha, o tema da história do Grupo de Bogotá- surgido na Universidade Santo Tomás- e a Filosofia latinoamericana, nas décadas de 1970 e 1980, por meio do comentário de autores e textos que tem feito a análise e a crítica do mesmo grupo. Para isso, utiliza as lições de verão de 1988 de Peter Sloterdijk, extraídas de seu texto Venir al mundo venir al lenguaje que aborda o tema do nascimento como uma "poética do começar".

Descrevem-se cinco momentos da vida do grupo situada no movimento da filosofia libertadora no continente e no país, assim o primeiro, o nascimento como desligamento de uma filosofia inócua e repetitiva; segundo, a abordagem das necessidades ou urgências do projeto nascente, terceiro, o surgimento criativo das iniciativas que deram lugar às instituições e mediações ainda presentes na vida da universidade e do país; o quarto momento da demora ou da pausa que se relaciona com a autocrítica; o quinto momento do trabalho de consolidação próximo da maturação. A través do texto aparece o papel decisivo desempenhado na formação do grupo pelos mestres da Filosofia da Libertação, especialmente o mestre Enrique Dussel.

Palavras-chave: Filosofia da libertação, Grupo de Bogotá, a priori do nascimento, poética de vir ao mundo, congresso. 


\section{Introducción}

Como es sabido, en la Edad Media la lectio inauguralis indicaba el inicio de las actividades académicas de una etapa o sesión de estudios, y de alguna manera marcaba el rumbo de las mismas. En cuanto elemento del método escolástico, implicaba comentarios de textos de autoridad en niveles de diversa profundidad hermenéutica. Dado que heredaba de la lectura el recitar los textos, incluidos los litúrgicos y bíblicos, al estilo de la salmodia, ofrecía diversas cadencias según los acentos que se quería dar a lo interpretado.

Pues bien, la Universidad Santo Tomás ha cumplido un importante papel desde las primeras décadas de su reapertura en 1965, la reflexión se abrió hacia el díalogo de saberes filosóficos en el continente, siendo hoy una muestra de lo que su acción ha generado y cultivado, el interés por los estudios de género, los estudios culturales y los estudios poscoloniales.

Se realizarán comentarios de textos de autoridad con cadencia que señala acentos. También con esos textos se hará un ejercicio de tejido, en memoria de los pensadores uitoto, una de las etnias de la región amazónica, cuando tejen sus canastos: "la palabra no solo es el hilo -fibra- con que se teje la trama del canasto, también va dando lugar al contenido: el saber mismo" (Urbina, 1992, p. 7). Este texto tiene la intención de volver palabra una parte de lo vivido, además poder entregarlo sin temor $\mathrm{y}$, a la vez, sin arrojo ni soberbia; como se narra un fragmento, porque esto es el testimonio de una experiencia, de ninguna manera la única versión de un proyecto, ni la verdad de una historia grupal. Solo un punto de vista ¿un "fracto", una cadencia, un matiz tal vez?

Lo hago en nombre de tantos que nunca han sido y tal vez nunca serán invitados a hablar, también como reconocimiento a quienes hicieron conmigo el camino, cualquiera haya sido la variante que cada cual tomó, ya que merecerían ser nombrados de modo personal.

Confieso que cuando fui invitada, una especie de conmoción interior hizo que me sintiera por momentos hermanada con esa criatura que reposa en un paraje de Villa de Leyva; "broncosaurio" le llaman, van los turistas a mirarla y exclaman: “iparece vivo!”. Pero como mientras respiremos podemos retomar las decisiones y sobreponernos a los miedos, me dije, ¿por qué no?”. 
Me propongo pues - con la salvedad de la mirada fragmentaria, teñida de los matices que da la memoria que repasa la vida- hacer una lectura del nacimiento y primeros desarrollos del proyecto o trabajo filosófico en clave Latinoamericana, cuyo principal referente ha sido lo que se conoció después como El Grupo de Bogotá, y hacerlo a la luz de las propuestas de un autor contemporáneo europeo -los que amamos Latinoamérica también estudiamos y aprendemos de los europeos- quien ha descrito con profundidad el acontecimiento que pocas veces los filósofos miran: el venir al mundo, nacer.

Me refiero a Peter Sloterdijk, en sus lecciones de verano en 1988 en la J.W.GoetheUniversität de Frankfurt, publicadas en Pre-Textos, como Venir al mundo, venir al lenguaje y que él ha denominado una "poética del parto".

Este texto clama por un espacio específico para los estudios de género y es uno de los atributos del mío, parir -no únicamente, por supuesto-. Por eso también va dedicado a las mujeres que en América y en otros continentes traen al mundo hijos con dolor y con dolor los ven crecer, vivir y a veces también desaparecer o morir. Y desmarcando la femineidad de la maternidad, además a quienes acompañan a otros a dar a luz, mundos, ideas, prácticas, gestos, grávidos de mejor pensar o vivir.

Son cinco los momentos descritos por Sloterdijk (2006) en esa "poética del comenzar", que se dan a partir del nacimiento y que denomina "a priori”, así:

1. "A priori del desligamiento de la madre", que a su vez significa liberación y apertura a un mundo nuevo e imprevisible.

2. "A priori de las urgencias", la llegada al mundo de las necesidades que a su vez da paso al dinamismo.

3. “A priori de las iniciativas", que marca los comienzos y el empuje de lo creativo.

4. "A priori de la posposición", cuando se hacen pausas, el tiempo de lo secundario, de lo que ocurre al margen.

5. “A priori del escenario", o de la arena donde se desenvuelven las acciones, se pone en juego el drama que sigue a todo comienzo. 
A la luz de esas lecciones intento asomarme a lo que, por lo menos en nuestro país, en nuestra Universidad, fue el parto de una apuesta por una filosofía que ha defendido su denominación y algo más, como Latinoamericana. Rememorar como nacimiento los comienzos de ese movimiento que nos ha traído hasta aquí, distinguiendo en su devenir los cinco momentos enunciados, que no se dieron siempre de modo lineal: porque como pasa en la vida, los acontecimientos se intercambian, se sobre ponen, con duración, intermitencias y patencias variables y hasta confusas.

No tengo la pretensión de hacer algo así como un relato fundacional de la historia de esta Universidad que va desde 1580, es decir 435 años siglos de tradición, de aportes y riqueza que no podrían resumirse en estas páginas. Nosotros no tenemos la tradición, sino es la tradición la que nos tiene a nosotros (Sloterdijk, 2006), porque como enseñó Gadamer: no es la historia la que nos pertenece, somos nosotros los que pertenecemos a la historia. Asumo más bien que "el individuo se malentiende a sí mismo al menos tanto como se comprende la relación que vive el yo consigo mismo, siempre está cubierta por una malla de auto iluminaciones y oscuridades" (Sloterdij, 2006, p. 44).

Para esta tarea acudiré a quienes han trazado juiciosamente la trayectoria del grupo, hecho su valoración y su crítica, especialmente el profesor Germán Marquinez Argote -fue mi maestro-; Roberto Salazar Ramos, su principal autocrítico -compañero de marcha en esa etapa-; Damián Pachón Soto -mi alumno hace unos pocos años- $y$ por supuesto, el querido profesor y amigo Santiago Castro-Gómez -quien viviera la efervescencia de ese momento como estudiante en la Facultad de Filosofía de la Universidad-. Ellos son fuentes autorizadas, voces entrañables presentes en diferentes momentos de mi vida.

Cabe mencionar que hasta en Wikipedia hay rastros de este grupo. Por cierto, figura que desde 1986 en adelante vendría su lenta agonía, iparece vivo!

Evitaré en lo posible las citas directas de ellos. Comentaré en esta lectio sus textos que son autoridad para todos. Finalmente en este ya largo a priori personal, quisiera que no se reduzca a la evocación nostálgica de momentos pasados, como recuerdos inocuos de algo que ya no tuviese validez y más bien sirva como una incitación a las generaciones jóvenes, a los que todavía andamos por el camino, a los que nunca oyeron hablar del tema los invito a "seguirlo", a iniciarlo y sostenerlo. 


\section{Cinco momentos en la poética del comenzar}

\section{El a priori del desligamiento y la liberación}

El primer elemento en esta pequeña poética del mundo, es algo que llamaré "a priori del parto". El desligamiento de todo singular de la comunión fetal con la madre. Sin embargo, el desligamiento tiene también un aspecto liberado trae consigo la ligazón o el apego de la nueva vida al mundo. Sucede en el apego a lo Abierto, a lo Imprevisible, a lo Incierto (Sloterdijk, 2006, pp. 103-105).

Era el año de 1975, 10 años después de la reapertura de la Universidad, estamos celebrando pues que la Facultad de Filosofía asumía oficialmente el proyecto de Filosofía Latinoamericana.

Las circunstancias del contexto colombiano y latinoamericano han sido presentadas por uno de los gestores del movimiento, el profesor Germán Marquínez Argote (1993), estas eran crisis de modelos económicos, sociales, inclusive religiosos y emergencia de otros; alusiones al socialismo, a la teología de la liberación, despunte o consolidación, depende de cómo se lo mire, del proceso de normalización de la filosofía, años de agitación en todos los órdenes.

Hace una semana, el profesor Marquínez dictó una conferencia en el seminario de investigación de la Fundación Xavier Zubiri sobre este mismo tema, cuyo manuscrito amablemente me envió. Y a propósito de ese año 75 -lo llamó annus horribilis- dice:

A imitación de lo ocurrido en Francia en 1968, grupos estudiantiles se tomaron las estancias universitarias, organizaban a toda hora asambleas generales, en las que proponían cambios radicales en la educación. Todavía recuerdo un acto multitudinario en el que se leyó un manifiesto proclamando que Colombia en una década sería comunista. Dicho documento fue introducido en un botellón, que fue enterrado al son de himnos revolucionarios en el viejo claustro universitario de la USTA, para que, desenterrado diez años después, diera testimonio del cumplimiento de la profecía en él contenida (Marquínez, 2015, p. 36). 
La Universidad no podía estar ajena a ese palpitar en medio del cuestionamiento a una academia donde primaba el universalismo abstracto, surge, como en un verdadero parto, la pregunta por el sentido y la función de la filosofía. Ella debía salir de sí, momento del desligamiento, y así ocurrió. Por una parte, en lo formal tuvo lugar la desescolarización del programa en la facultad. Y por otra parte, en lo vital, comenzaba a llegar al país la apuesta por un filosofar de orientación latinoamericana que hoy nos tiene reunidos ¡alerta, a muchos todavía convencidos!

Era la ruptura del vínculo maternal, desprendimiento y apertura a otro mundo. No es casual que los gestores de ese primer aliento hubieran venido de Europa, la mayoría de España, y leyeran lo que alentaba en el interior de profesores y estudiantes colombianos.

Sin fracturas, al menos al comienzo, surge entonces con la frescura y el empuje de una vida que empieza este proyecto que el Padre Joaquín Zabalza Iriarte, alma y nervio de este inicio, describía así (como se cito en Marquínez, 1993).

Este nuevo estilo de filosofar se ha ido abriendo camino entre nosotros, ello se debe sin duda, por una parte al diario e inquieto diálogo informal entre los profesores de filosofía de la Universidad y por otra a los frecuentes seminarios organizados por el Centro de Enseñanza Desescolarizada (p. 365).

Es decir, lo anterior alude a la vida universitaria. Señalo dos aspectos de ese nacimiento, además de los destacados en varios textos; el primero, la convocatoria que nos hacía el decano -P. Zabalza- a decir nuestra palabra, de diversas maneras, sobre el tema. En un memorando de 1978 que conservo como parte de mis memorias escribió: sírvanse señores profesores, señores estudiantes de la facultad, entregarme a la mayor brevedad una cuartilla al menos de lo que para cada uno es una Filosofía latinoamericana. Entonces, había que responderle. Por encargo suyo las respuestas fueron transcritas y recopiladas más tarde, como guía y también memoria de la facultad.

El segundo, la circunstancia conocida como "normalización” de la filosofía en el país, juiciosamente estudiada por el profesor Marquínez, Rubén Sierra Mejía, recientemente Damián Pachón Soto, entre otros, situada a partir de esa década de los 70. 
Convocarnos a decir nuestra palabra era la manera de conectar el desligamiento con la inserción en lo Abierto, lo imprevisible, lo incierto. Porque, para seguir el discurso de Sloterdijk:

Todo hombre encarna una sílaba, una suerte de excrecencia única e intransferible de consonantes y vocales, una sílaba viva que va de camino a la palabra, al texto. Lo que ayuda a estas sílabas vivas y ocultas para sí mismas a encontrar la huella de su propio sonido sería la escritura. (Sloterdijk, 2006, p.18).

Tal vez esa es la razón por la cual encontramos en los anaqueles de los antiguos profesores o estudiantes de esa época, una serie de títulos de obras que abrieron la perspectiva latinoamericana en la Facultad de Filosofía de la USTA, y llegaron a regiones remotas con la mediación de la educación a distancia. Porque "el lenguaje es la instancia que nos da propiamente el mundo, la que abre ese paisaje extático en el que los hombres se mantienen" (Sloterdijk, 2006, p. 106).

Esas primeras obras, los textos, los discursos, que fueron y son sometidos aún hoy a feroces y seguramente jUSTAs críticas, eran la concreción del momento liberador de ese primer "a priori" de nuestro parto.

Y fuimos nombrados como "grupo", y luego más tarde enjuiciados, también valorados y ahora hasta estudiados como tal. Desconozco si esa es la denominación que correspondía a un entramado plural de edades, tendencias, cadencias, que vivimos la experiencia sin llamarnos de ninguna manera. Tal como no se tiene la facultad de ponerse el nombre cuando se llega al mundo, pues otros lo hacen por uno.

Quienes han estudiado el tema, tanto Germán Marquínez Argote como Roberto Salazar Ramos, reconocen la existencia de dos supuestos comunes que, según ellos, nos acompañaron largamente y han servido de referencia para esos análisis:

- América Latina como horizonte concreto y situado de nuestras reflexiones filosóficas.

- La intencionalidad liberadora de la filosofía al interior de ese horizonte.

Elementos puntuales, de relación, discusión y diferenciación. Santiago Castro-Gómez (2011), ha mostrado cómo los miembros del grupo "no distinguieron muy bien entre las dos tradiciones de filosofía latinoamericana que se desplegaron el siglo pasado: 
el historicismo y el liberacionismo y [el Grupo] tendió a subsumir las dos bajo una única etiqueta: la filosofía latinoamericana de la liberación" (p. 13). Y como pasa en todo nacimiento, desligarse era también abrirse a lo nuevo.

El primer Congreso Internacional de Filosofía Latinoamericana (1980) se inauguró con un cupo total (más de 400 personas) y con la presencia entusiasta y participativa de profesores, estudiantes, asistentes de todas las regiones del país y la presencia de ilustres filósofos latinoamericanos de más de doce países (Argentina, Chile, Brasil, Uruguay, Perú, Ecuador, Venezuela, Panamá, Costa Rica, Honduras, México, República Dominicana). Una rápida lista con sus nombres nos permite hoy tomar consciencia de ese momento: Leopoldo Zea, María Luisa Rivara de Tuesta, Hilton Japiassu O. P., Samuel Guerra Bravo, Constanza Macondes César, Juan Mora Rubio, Enrique Hernández, Eugenio Lakatos, Hugo Assman, Daniel Vidart, Enrique Leff, Enrique Dussel, Diego Gracia Guillén, Germán Marquínez, Alberto Parisi, Guillermo Hoyos, sin nombrar los demás de la casa.

Jornadas nocturnas precedieron la apertura, profesores y estudiantes armábamos artesanalmente las carpetas con el entusiasmo de un comienzo presentido y muy esperado. $Y$ en el cierre, nuevamente, las palabras del decano citadas varias veces en otros contextos, que señalaban que el término de ese congreso no era solo su cumplimiento sino algo más importante: porvenir, apertura al futuro porque:

Filosofía no es el sUSTAntivo y Latinoamericana el adjetivo, el accidente que se quita o se pone. (...) Filosofía y Latinoamérica son dos sUSTAntivos incompletos, dos principios inconclusos de un ser cuya unión sUSTAncial, sUSTAntiva, posibilita la única y auténtica filosofía (Zabalza, 1981, p. 21).

¡Qué importante recordar esto hoy! Un apasionado comienzo: ¿acriticidad romántica?, ¿veleidades de mentes juveniles?, ¿palabra que despierta?, ¿alteridad que busca su puesto en la historia? La lectio, debe llevar a la questio.

\section{El a priori de la urgencia, el mundo de la necesidad}

El a priori del parto debe complementarse con el a priori de la urgencia. Apenas hemos arribado a lo abierto, y y comienza nuestro apego al mundo de tal forma que estamos transidos de urgencia. La necesidad no solo enseña, como todo el mundo 
sabe, a rezar, sino también a ser de este mundo. Aprendemos el oficio de la vida cuando lo inaplazable necesario nos arranca respuestas constitutivas del mundo; gracias a ellas nos convertimos en seres archipoetas a la vez que protoprosaicos. Al dictado de las urgencias redactamos nuestros textos basales del mundo

(Sloterdijk, 2006, p. 108).

No cabe duda, asumido el proyecto llegaron urgencias en múltiples aspectos. Más allá de atender las demandas de calidad de los mismos estudiantes y de una facultad que reposaba cómodamente en una docencia inocua, a veces repetitiva, recibíamos el urgente llamado de una filosofía distinta, donde hubiera espacio para las necesidades del "otro como otro"; filosofía que ya en Latinoamérica había abierto un camino hacia el compromiso por la liberación y ante ello surgieron respuestas no solo poéticas, algunas "románticas" como han sido calificadas después, sino también avances prosaicos, como veremos en el tercer momento de las iniciativas. Urgencia de apoyarnos en maestros, urgencia de mirarnos desde la tradición propia, etc.

Y maestro fue ante todo nuestro querido profesor, Enrique Dussel, a quien la Universidad le ha dado su debido reconocimiento, también todos los filósofos y maestros de la Filosofía de la Liberación, así como los de casa, entre ellos como lo hemos dicho, el profesor Germán Marquínez Argote, con su lectura crítica de Descartes y Heidegger, quienes nos alimentaron en nuestras urgencias y en la lectura de nuestras necesidades.

Es interesante ver cómo en este mismo texto que me sirve de referencia y a propósito del a priori de las urgencias y la función de las necesidades, Sloterdijk (2006) se refiere a Marx y a Heidegger:

Los dos descubren también en la realidad social de los hombres los vestigios de lo urgente e ineludible la huella del cuidado y del impulso. Los dos interpretan al ser humano antes que nada como aquella criatura cuyo venir-al-mundo tiene que atravesar el paso estrecho de lo urgente. Marx interpreta la dimensión de la urgencia a través del trabajo humano entendido como producción y apropiación. Heidegger, por su parte, la lee como una suerte de modelo de lo que encomienda a la existencia la posibilidad de cuidarse (p. 110). 
Marx y Heidegger, dos autores que para el Grupo fueron determinantes. De pronto no fue una coincidencia. Aquí, la memoria es selectiva, toma partido, tal vez quiere ser justa y no siempre lo logra.

Constituida la Asociación de Filosofía Latinoamericana en Colombia - AFIL, en Bogotá, especialmente con los integrantes del Grupo y los demás interesados, aparece entre otras necesidades, la de estudiar a Marx, herramienta y mediación de la Filosofía de la Liberación, como en buenos debates se ha señalado; así que dedicábamos nuestras tardes del sábado a clarificar un materialismo que nos hacía entender lo que significaba la economía -condición para bien entender la económica en Dussel- puesto que implicaba apropiarse de las condiciones en que se produce y reproduce la vida humana, esto es, la vida del "otro" que nos marcaba y nos hacía sensibles a toda necesidad, como lo estábamos aprendiendo de los filósofos de la liberación.

Y en cuanto a Heidegger, textos como el de Metafísica en América Latina del profesor Marquínez, entre otros estudios y seminarios, sin lugar a duda marcaron también derroteros, enrutaron trabajos de grado, etc.

Dos hermenéuticas en ese momento, según Sloterdijk (2006):

La hermenéutica del trabajo [de Marx] como pasaje del reino de la necesidad al de la libertad. [La hermenéutica de Heidegger] aborda la resolución acerca de lo pesado como tránsito que media la expropiación y el desasimiento. El ser humano marxiano ha de forjarse echando abajo el peso del mundo pasando por las astucias de la producción; el ser humano heideggeriano tiene que endurecerse para encontrar el desasimiento heroico en el ojo del huracán. En ambos casos el hombre tiene que hacer algo de sí mismo si no quiere caer o verse reducido a la miseria (pp. 110-111).

Por supuesto otros énfasis se hicieron presentes en los textos escritos en esa circunstancia, como lo muestran los trabajos del profesor Jaime Rubio Angulo -quien nos abandonó temprano-, cuya propuesta insistió siempre en la importancia de la resignificación de la tradición mediante la hermenéutica como método ineludible para pensar la liberación como sentido, al interior de la Filosofía Latinoamericana. Fue el primero en escribir una Introducción a la Filosofía Latinoamericana desde 
una óptica diferente. Destaco a Marx y Heidegger porque a pesar de sus diferencias aparentemente irreconciliables:

Se presentan como los dos pensadores más relevantes de lo necesario para el hombre, y ambos pueden ser considerados como los impulsores de las teorías determinantes de lo político, habida cuenta de que comprenden la esencia del poder como presencia en el espacio de la decisión sobre lo urgente (Sloterdijk, 2006, p. 110).

Reitero, es relevante que a la luz de ambos autores, camináramos en esa década, amén de, cómo lo ha señalado el profesor Castro-Gómez (2015): el socialismo cristiano, criticado por la forma como fue acogido, afirma él, como espejismo en cuanto a estudio de la realidad latinoamericana en ese momento. Pero socialismo que de alguna manera también estaba presente en los trabajos y algunas decisiones del grupo.

Un texto del Teólogo de la Liberación Hugo Assman, en su ponencia en ese primer congreso, evoca el espíritu vivido en esos momentos:

El hombre es su corporalidad, sus condiciones materiales de existencia; no se puede hablar del hombre sin hablar de su rostro. La vida corporal y material es el único valor absoluto para el hombre. Todos los demás valores derivan su fuerza hacia la trascendencia y hacia lo absoluto a partir de esta vida corporal y material. Yo opto por la ruptura con el sistema que es incapaz de satisfacer las necesidades básicas del hombre, y a partir de allí, y en la lucha paso a paso, con metas estratégicas claras, pero paso a paso también concretos y pacientes, iremos construyendo una sociedad en la cual los hombres puedan ser más hermanos y donde puedan también experimentar y realmente vivir algo que les permita una experiencia espiritual, a partir de la alegría del trabajo, de la alegría del pan, de la alegría del amor, de la alegría del toque de la piel, de la corporeidad y de la erótica y de las canciones de las danzas. No veo ninguna otra perspectiva para una experiencia espiritual donde se pueda nombrar el misterio (Assman, 1981, pp. 169-175).

Pero no solo nos movieron las urgencias interiores y pensantes, también las institucionales, las pedagógicas, las personales. Esta es solamente una mirada parcial, porque las lecturas no pueden ser unívocas. El mismo padre Zabalza, a propósito de una visión analógica de la historia escribía: 
De ella dependen en parte, nuestra propia autocomprensión del presente y nuestra adecuada proyección hacia el futuro. Analógicamente entendidas, ni la filosofía de la historia es una filosofía de la "histeria", ni la historia de las ideas es mera "arqueología" de las ideas (Zabalza, 1993, p. 22).

\section{El a priori de la iniciativa}

A partir de las urgencias nacen los mandatos con nombre de necesidad, y a su vez se vuelven nuevamente urgencias. En todo caso el hombre ha de hacer algo de sí mismo. Es necesaria la transición de la urgencia a la iniciativa. Dice Sloterdijk (2006) que:

El concepto de esta transición cargado de fatalidad en la era moderna, no es otro que el de revolución. En el centro mismo de la necesidad está en marcha un imperativo orientado a atajar la necesidad, en la poética del venir-al-mundo aún tiene que suponerse un tercer momento: el a priori de la iniciativa (p. 110).

\section{¿Hacia dónde?}

"Los seres humanos para venir al mundo tienen que comenzar algo: sin un comienzo propio no hay mundo, este "algo" no es nada arbitrario, significa de entrada un resuelto comenzar con uno mismo, literalmente comenzarse" (Sloterdijk, 2006, p. 112). A propósito de "revolución": fuimos señalados como revolucionarios, tanto como de “¡mamertos!”.

Para describir este comienzo, Sloterdijk (2006) utiliza como es su estilo, una serie de metáforas que hacen el efecto de trasladarnos a múltiples vivencias, sentimientos, etc., del proceso que estoy narrando. Flujos de sentimiento y razón que evocan, me atrevo a llamarlo así, esa "razón poética” de María Zambrano.

Comenzarse comenta él: como una bomba que se activa, una obra aún no interpretada, una señal de salida para una carrera que empieza, una puerta que se abre para dar paso a un lugar que nunca ha existido, un arma a la que se le quita el seguro, en fin, en sus palabras, un acto de autoencendido político existencial.

Se dieron en el Grupo muchas formas de vivirlo y de trascenderlo. Por eso, algunos tomaron otros caminos o desde dentro vieron horizontes distintos y los siguieron; como 
Roberto Salazar Ramos quien inició a la luz de Foucault, la arqueología del mismo grupo; otros a través incluso del rechazo, como en el caso de Santiago Castro-Gómez, atravesaron la propuesta para encontrar su propio y, en su caso, brillante camino.

Interpelado por un estudiante de Filosofía en el año 2011 acerca de la influencia del "Grupo de Bogotá", Santiago Castro respondió que era bastante, ya que le mostró el camino para su trabajo y, además, la manera equivocada de recorrerlo. Y comentó que si bien rechazaba la filosofía de la liberación le interesó mucho la historia de las ideas y de ahí su fascinación por el proyecto de trazar una historia de nuestras tradiciones intelectuales.

Otros seguimos creyendo en el proyecto y consideramos que nuevas generaciones pueden buscar su camino sin perder de vista América y todos los pueblos que de alguna manera están hermanados en su destino y en sus oportunidades. Esta misma idea animó en su momento varias acciones; entre 1975 y 1980 surgen y se consolidan iniciativas que hoy, 40 años más tarde -son casi cincuenta, con C y ojalá también sin cuenta con S-, se pueden ver en la vida de la Universidad, en la academia, en proyectos cristalizados en el país y más allá de él.

Iniciativas que comenzaron desde que al preguntar por el sentido del filosofar se intentó -la expresión es del profesor Marquínez y desde entonces se repite muchas veces y no se entiende- que la filosofía fuese raizalmente situada pero terminalmente universal, y por ello se decidió que la Facultad de Filosofía abriera sus puertas y se desescolarizara, adelantando un proceso que más tarde fue implementado por el Gobierno nacional, pero que en nuestro caso buscaba llegar a todos los rincones de Colombia donde fuera posible. Por eso los profesores viajaban por todo el país, dictaban seminarios, escribían manuales y textos, siempre marcados con las dos señales que convocaban: Liberación y Latinoamérica.

A continuación una anécdota, entre muchas. Un grupo de estudiantes que estaban citados a sus exámenes teniendo que desplazarse a Bogotá desde la región del Caquetá, que después fue lugar de los primeros malogrados diálogos de paz, no pudieron llegar a tiempo, pues habían sido retenidos por un grupo, de los entonces sin desmovilizar, guerrilleros del movimiento M-19. Cuando se les preguntó cómo sortearon el citado retén, respondieron que mostrando las carátulas de los textos Ética de la Liberación en América Latina y Educación y liberación en América Latina. 
En 1980 se institucionalizaron estos Congresos Internacionales de Filosofía Latinoamericana que por muchos años fueron sostenidos por la asistencia de los estudiantes y profesores del Centro de Enseñanza Desescolarizada, CED hoy VUAD y por ello con la presencia activa y vibrante de la región.

Y como cuando venimos al mundo estamos marcados por la impronta familiar, el decano padre Zabalza (1981), señalaba que este aporte era el fruto de un trabajo lúcido y apasionado, porque:

\begin{abstract}
Ustedes perdonen que, los profesores y estudiantes de esta Facultad no puedan ser de otra manera, porque hubo en el siglo XIII un filósofo de etimología napolitana y rigor alemán, cuyo nombre preside esta Universidad. No quiso ser y no fue platónico sino más bien experimentalista aristotélico, analítico y sintético y realista. No quiso ser y no fue feudal sino comunalista, "otro que el sistema". No quiso ser y no fue monje contemplativo, sino fraile peregrino por los caminos de Europa, por los suburbios de las ciudades, por los claustros universitarios: predicador, hablador, contestatario junto a los herejes de su tiempo (p. 21).
\end{abstract}

Es una nota al margen para las nuevas generaciones de tomasinos que nos acompañan, y una evocación para todos aquellos a quienes ese comienzo convocó, desde distintos espacios de la academia y de la vida universitaria, a motivarse para pensar nuestra realidad, con seriedad y con pasión. Como señaló el decano en la clausura:

Ello quiere decir que la filosofía no se acaba como algunos augures pesimistas vaticinan y que su convocatoria obtendrá contestación positiva en Colombia, en la Patagonia, en Choluca siempre y cuando el hombre latinoamericano encuentre en ella una dimensión de sentido y esperanza (Zabalza, 1981, p. 21).

En 1977 se había creado el Programa de Maestría en Filosofía Latinoamericana. Contando con numerosas promociones de estudiantes de todas las regiones del país y algunos de otros países latinoamericanos, formados en la responsabilidad social y ética por los proyectos de nuestros pueblos. Su presencia ha sido activa en estos mismos congresos, en eventos, dentro y fuera del país, y en sus regiones, con movimientos, escuelas de pensamiento, publicaciones, líneas pedagógicas, donde la filosofía sigue estando a la escucha de las necesidades de la realidad y a los giros que los estados de poscolonialidad les exigen. 
Durante la preparación del primer congreso, en 1979, se fundó la revista Cuadernos de Filosofía Latinoamericana; 36 años, 112 números de publicación ininterrumpida. En 1982, el Centro de Investigaciones de la Facultad, orientado a recuperar nuestra tradición pensante; su campo de trabajo inicial, la historia de las ideas; hoy abierto a otras perspectivas y nuevos métodos de investigación.

Con la transcripción y traducción de textos latinos de manuscritos filosóficos coloniales, se inició la colección Biblioteca colombiana de filosofía (hasta con hoy 30 volúmenes). Su primer director y alma de la misma como de todo nuestro trabajo posterior, el maestro Daniel Herrera Restrepo. Inmenso reconocimiento a él.

Porque nacer no es solamente desligarse, también es traer consigo los genes, la herencia de cuanto nos ha engendrado; así no todo nos llegó desde fuera, como lo advierten los estudiosos de la poscolonialidad y decolonialidad; invitados y algunos presentes en este evento.

Hoy, con Mignolo (2009):

La tarea a futuro no es tanto pelear con los molinos de viento llamados "capitalismo global" sino con las intrincadas fases, esferas y dominios en los que hoy la matriz colonial de poder está en disputa en un orden mundial policéntrico (p. 24).

Volviendo al comienzo, nacieron también las editoriales pioneras en recoger esa memoria. Primero Editorial Nueva América, luego Editorial El Búho. Desde ellas también, nuestro vínculo con las regiones se hizo fuerte y alimentó el proyecto. Hoy, por su constancia, siguen permitiendo que el pensamiento latinoamericano esté al alcance de profesores, autores y estudiantes. Así que el trabajo no se encerró en los claustros de la Universidad ¡como en los primeros años de la infancia, hicimos ruido!

A propósito de las regiones, ¡un sentido reconocimiento para los grupos de profesores, estudiantes y filósofos, de ellas, donde la propuesta de pensarnos ha dado tantos frutos! (La región Caribe, Cauca, los santanderes, Huila y Tolima, y todas las demás).

El primer proyecto educativo, considerado como tal en esta Universidad, cuando aún no se hablaba mucho del tema, fue obra del trabajo colectivo de los profesores del Centro de Enseñanza Desescolarizada - CED, liderados por su director, el doctor Luis José González Álvarez. Dicho proyecto incluía la propuesta del impacto en el 
desarrollo de las comunidades locales. Por ello las asignaturas de las licenciaturas en filosofía y educación a distancia debían estar vinculadas a algún proyecto social de la región. Desde entonces tuvimos la convicción de la necesidad de hacer praxis la palabra. Como un ejemplo entre muchos, en Cúcuta, las madres estudiantes de las licenciaturas de Primaria y Promoción de la Comunidad y Preescolar y Promoción de la Familia, crearon un célebre kínder u hogar donde se turnaban para cuidar sus hijos y estudiar. Ese hogar, por cierto, se llamó: “Tomasito”.

Como grupo que inicia un nuevo camino, asumimos que no empezábamos en el vacío; nos insertamos en una corriente presente desde hacía casi una década, especialmente en Argentina, México, Perú y Ecuador, por lo mismo nos propusimos aprender de los que reconocíamos como maestros, el principal de ellos, como lo hemos señalado, nuestro profesor Enrique Dussel.

En el imaginario de quienes hacen la historia de este periodo, el colectivo bebió de esa fuente, se alimentó de ella, fortaleció sus intencionalidades y en ello encontró su fortaleza y también, como veremos, algunas de sus debilidades.

Cuando con entusiasmo aprendemos, qué duda cabe del peligro de aferrarnos a un esquema, a un vocabulario, a un perfil de lo aprendido, no siempre de manera crítica. Fuimos por supuesto dusselianos en muchos sentidos -ojalá lo siguiéramos siendo en otros- pero no se piense que la experiencia se dio tan acríticamente como en algunos contextos se narra.

En la revista Cuadernos de Filosofía Latinoamericana n. ${ }^{\circ} 12$, de 1982, apareció reimpreso un artículo de Jaime Rubio Angulo, La filosofía en Colombia, una crisis que da qué pensar, publicado por primera vez en 1977, que provocó de parte del maestro Dussel una carta al director (de la revista), en noviembre de 1982, donde replica pormenorizadamente juicios del citado artículo, que consideraba erróneos. Especialmente replica las alusiones de Jaime a la cercanía de los filósofos de la liberación con el peronismo, y su inclinación hacia el marxismo y el señalamiento hecho a la naciente Filosofía de la Liberación por no dar sitio preponderante a la tradición. A todo ello responde Dussel puntualmente. Como el director de la revista le solicitara a Rubio una aclaración, este escribió, "nada debo aclarar. No hay una sola frase de mi trabajo que desee cambiar o de la que quiera retractarme"; y cita en esta nota aclaratoria, que por supuesto la revista publicó en el n.o 14 de 1983, la siguiente frase de Francis Bacon: 
Los discípulos deben a sus maestros solo una fe temporal y una suspensión del propio juicio hasta tanto no han recibido una instrucción completa, pero no una dimisión absoluta ni un cautiverio perpetuo de su mente. Así, pues dejemos que los grandes autores reciban el tributo correspondiente, sin que el tiempo, que es el autor de todos los autores se vea privado del suyo, el cual consiste en avanzar ininterrumpidamente en el descubrimiento de la verdad (pp. 97-100).

En ese mismo número de la revista, Roberto Salazar Ramos, integrante del Grupo, publica un artículo, De la Filosofía Latinoamericana a la filosofía de la liberación. Radiografía de una crisis, donde inicia esa tarea, la radiografía, que anunciaba su posterior y valiosa autocrítica. Hablaba de respuestas puramente afectivas teñidas de irracionalismo, politización, formalismo, espiritualismo en la mirada de la historia, antropologías donde se deslizaba el populismo, temáticas en las que la cultura, la historia, la literatura, mostraron cómo no, desfases entre la reflexión y la vida.

Señalaba que, categorías como: pueblo, nación, praxis, liberación, historia, lucha de clases, "deben someterse a profundas críticas, si no queremos caer, como en algunos casos se ha hecho, en un lenguaje panfletario y estereotipado. Esto ha valido el reproche de psicologismo, de irracionalismo y afanes inmediatistas" (Salazar, 1983, p. 91). Vale decir que en las editoriales citadas, algunos miembros del grupo escribimos artículos sobre esos temas.

Es una muestra solamente de cómo ese, “a priori de lo urgente”, no se vivió de modo tan inocente o acríticamente como se ha leído pero sí significó lo que comenta Sloterdijk (2006), refiriéndose a Heidegger: “el apriori de la iniciativa, por así decirlo, se abre hacia atrás. Comenzarse puede significar: abrirse retrospectivamente a las voces y huellas de otro comienzo por medio de la percepción ilimitada del haber-sido efectivo" (p. 117). Algún filósofo norteamericano llamó a esto “idealismo de la nostalgia”.

\section{El a priori de la posposición}

Iniciado el movimiento, después de las urgencias, las iniciativas importantes precisamente por ser difíciles exigen más tiempo y terminan siendo de algún modo pospuestas. Llega el momento de "la demora", de la "pausa”. Así: 
En "el a priori de la posposición" que también podría llamarse el principio de demora, aparecen preludios a los asuntos principales, y dichos dominios se pueden animar, habitar y administrar. Podría decirse que gran parte del radio de acción del arte de la vida hace referencia al arte de las cosas secundarias, una buena parte de la vida real no se desarrolla en el campo de juego, sino en el margen, no bajo un plan fundamental sino en la pausa. En estos preludios, el tono discreto es el dominante, los héroes son los personajes secundarios (Sloterdijk, 2006, p. 120).

Por ello, en esta lectio, hemos de rendir tributo a compañeros, discípulos, maestros, exalumnos, trabajadores; a todos cuantos silenciosamente le apostaron a este proyecto creyendo en un futuro, trabajando en la pausa, al margen, posponiendo intereses personales sin destacadas remuneraciones, ni condecoraciones, pero ex-poniéndose, como decía el padre Zabalza en el cierre del primer congreso, sin temor a ensuciarse la manos. Homenaje a toda la gente que en ese margen eran, en palabras de Salazar (1981), “pioneros de una reflexión filosófica que en el caso colombiano, se juega su ser y su existir por la vida y la esperanza de quienes en una historia necrófila, hemos sido confinados al no ser y a la sinrazón” (p. 13). Juicio que esperemos sea solo del tiempo pasado.

¿Qué significó este cuarto momento o principio de la demora y la posposición? Hay diferentes maneras de mirarlo.

¿Una pausa en la intencionalidad política intelectual de la Universidad, que atravesada por avatares de su propia historia, viviera años que no tenemos derecho a juzgar, no al menos en su dimensión de historia interna?

¿Pausa en el empuje por la fatiga que sigue a todo gran esfuerzo; en las capacidades de un grupo en el cual también otras historias personales, intervienen, actúan al margen, interfieren-detienen? Cada uno tendrá la manera de contar la experiencia. Cada relato tiene su cadencia.

Yo, relaciono este momento con la autocrítica, como pausa que al interior del mismo grupo surgió tempranamente y se hizo patente y pública en el IV Congreso Internacional de Filosofía Latinoamericana en 1986. 
Después del primer congreso, ese entusiasmo, en palabras de Marquínez, eclosionó en un público enfervorecido, levantó críticas por un pretendido modelo de filosofar situado y liberador; aceptación o rechazo apoyados en razones tanto como en malentendidos. Vino un II Congreso sobre Historia de las ideas en América Latina, el III sobre Identidad cultural, con aportes más sosegados, razonables y razonados, hasta que en el IV se planteó el ser o no ser de la filosofía en Colombia.

Un año antes, en 1985, la Universidad había organizado el 28 de mayo un EncuentroHomenaje con motivo de la presentación del texto Filosofía en Colombia, bibliografía del siglo XX, preparada por el Centro de Investigaciones de la Facultad con la dirección de la profesora Gloria Isabel Reyes, hoy su directora, quien con los estudiantes realizó numerosas entrevistas a los autores, de manera que ellos mismos validaron la publicación; algunos hasta solicitaron ser incluidos en ella. Homenaje a los filósofos colombianos llamados de la normalización. Encuentro de estudiantes, profesores y maestros de distintas facultades y departamentos de Filosofía del país; distintas generaciones, tradiciones y orientaciones.

Esta diversidad [anotaba el Decano] constituye el "conflicto" mismo que es la filosofía, fenómeno escandaloso para el que mira desde fuera los resultados de su historia sin percibir en el proceso una voluntad de verdad que penosamente se despliega y que continuamente se critica a sí misma, depurándose (Zabalza, 1986, p. 7).

Homenaje a los normalizadores, dos de ellos, para entonces ya desaparecidos, Cayetano Betancur y Luis Eduardo Nieto Arteta y los demás presentes allí: Abel Naranjo Villegas, Jaime Vélez Sáenz, Danilo Cruz Vélez, Rafael Carrillo Luquez, Ramón Pérez Mantilla y Daniel Herrera Restrepo.

Después de este homenaje, en 1986, tuvo lugar el citado IV Congreso de la Autocrítica, también durante el mismo, la presentación oficial de la Biblioteca Colombiana de Filosofía, en sus tres primeros títulos con la obra de Danilo Cruz Vélez, Rafael Carrillo y el profesor Daniel Herrera.

¿Cuál es el núcleo de la crítica que nos permitió hacer la pausa?

El acápite de la ponencia del profesor Salazar Ramos en dicho congreso, nos da la clave: 
No se puede hablar en cualquier época de cualquier cosa, no es fácil decir algo nuevo; no basta con abrir los ojos, con prestar atención, o con adquirir conciencia, para que se iluminen al punto nuevos objetos, y que al ras del suelo lancen su primer resplandor (Foucault, 1974, p. 73).

Ya a la luz de Foucault, Roberto abría otros caminos para la tarea; era como el ícono de lo que también había propuesto el padre Zabalza en el citado homenaje cuando afirmaba que "día a día seguiremos inquiriendo por presencias colombianas, día a día seguiremos indagando naturaleza latinoamericana y la no búsqueda de esencias históricas universales" (Zabalza, 1986, p. 9).

Roberto contextualizó nuestro trabajo, situándolo en el movimiento de la Filosofía Latinoamericana y toda la circunstancia del continente en los distintos aspectos, y también en los debates de los maestros Salazar, Zea, Dussel, que nos habían llevado a ubicarnos en la filosofía como opción de liberación. Él, que había hecho parte del grupo desde el 78, se propuso hacer una autocrítica a los equívocos vividos y reconceptualizar, enderezar si era necesario, el camino. Señaló primero las dificultades, nacidas en parte de las mismas urgencias de la docencia y del desarrollo que tomaron las conceptualizaciones. Partió del convencimiento de que ni había un discurso ideal arquetípico que nos hubiera guiado, pero tampoco lo que estábamos haciendo era un juego secundario de opiniones.

A partir del análisis de los textos de Jaime Rubio Angulo, hizo una radiografía de cómo operaba en cuanto enseñábamos y estudiábamos, un mismo esquema que calificó como mecanicista, tomado de la sociología de la dependencia y trabajado en términos de filosofía, un esquema en el cual el telos de la liberación cerraba una especie de círculo. Así analizó uno a uno los principales aportes de autores y trabajos y la manera como para el abordaje crítico de la tradición filosófica y la recuperación de la tradición clásica asumimos la intencionalidad dusseliana, su esquema y su búsqueda. Análisis que sirvió de base para el desarrollo de la concepción de la labor destructiva del arkhé de la ontología moderna y de sus subsiguientes manifestaciones.

A decir verdad, por temas como la posible ruptura y superación del horizonte de la subjetividad moderna, la apertura a la metafísica situada en el otro como exterioridad, etc., pasamos no siempre fácilmente, entre dudas, contradicciones y repeticiones. 
Con honestidad a toda prueba, valiéndose de sus propios textos -el primero escrito en 1976 en la Universidad San Buenaventura donde fue formado-, Roberto mostró cómo a pesar de que sus artículos buscaban:

Articularse como una ruptura en el plano de lo epistemológico, el esquema utilizado correspondía de una u otra manera, al esquematismo de la geopolítica contemporánea, partiendo para su actual estructuración, del expansionismo europeo del siglo XV. El esquema, se convirtió. [Concluye] de algún modo en el lugar común y en un comodín sospechoso. (...) Vislumbramos la simplicidad del esquema y sin embargo, lo utilizamos literalmente, en muchos casos (Salazar, 1986, p. 59).

Dicho así, aparece como un proceso con decurso lineal. Hubo sin embargo debate entre los mismos autores. Caminos metodológicos diferentes: la hermenéutica, la metafísica, la antropología del personalismo cristiano.

Germán Marquínez Argote había pedido conceptualizar con rigor; lo mismo hizo el doctor Daniel Herrera Restrepo en Escritos fenomenológicos, de la Biblioteca Colombiana de Filosofía, y reiteró en la recordada lectio inauguralis en la Facultad de Filosofía donde invitó a hacer la crítica de la razón latinoamericana (como bien se sabe esa lectio inspiró el tema y el título del libro clásico de Santiago Castro-Gómez, reeditado en el 2011). Preocupación que a lo mejor sigue siendo una tarea pendiente: hacer el "análisis de los conceptos y la elucidación de sus categorías orgánicas. Pero desde el interior mismo de la racionalidad filosófica” (Salazar, 1986, p. 67), dicha cita es de 29 años atrás.

El profesor Roberto concluyó, a propósito de los dos escenarios, pedagógico y filosófico, que el primero fue dinamizador del segundo. Los dos vinculados a sus aciertos y también a sus fallas.

Una determinada forma de activismo institucional nos debe ser imputada. En realidad, un mayor espacio teórico interno hubiese desencadenado discusiones muy fructíferas entre los miembros del Grupo. En cambio, las urgencias pedagógicas y la labor docente desequilibraron el énfasis en la actividad teórica (Salazar, 1986, p. 80). 
Y, ¡en ese tiempo no había urgencias de acreditaciones! Casi 30 años después me pregunto por la actualidad de ese juicio. Se habló de fases diversas que fueron del romanticismo retórico y maniqueo, al intercambio y el diálogo con otros representantes de la Filosofía Latinoamericana y con otras tendencias; del reconocimiento de los equívocos y los aciertos, a la necesidad de la fase creativa. "Porque nos hemos equivocado en muchas cosas, es por lo que permanecemos aún en el campo de la filosofía" (Salazar, 1986, p. 81).

¡Cuántos ecos, comentarios y anécdotas siguieron a esa ponencia y ese congreso! Este momento, largo momento de autocrítica que he asimilado a una posposición, o pausa, frente a dificultades internas y externas, ¿cumplió su cometido? ¿Fuimos capaces de ir más allá de los equívocos, apoyados en los aciertos y avanzar abriendo camino a otras miradas, otras maneras de articular la filosofía con la realidad tan urgida de cambio?

Seguramente en algunos aspectos sí y por eso seguimos vivos -no solo parece vivo, no morimos, como Juan Preciado de Rulfo, por fuerza de los susurros y el miedo-, seguramente en otros aspectos no, y por el camino quedaron posibilidades, intentos silenciosos, proyectos y hasta historias personales que se frustraron, salieron lastimadas o ignoradas. El precio que se paga por vivir.

\section{A priori del escenario o de la arena}

Cuando el a priori de la postergación ha cumplido su cometido surge el quinto elemento de nuestra poética del mundo en liza. Yo lo llamaré el a priori del escenario,

O por decirlo de otro modo: el a priori de la arena o dramático (Sloterdijk, 1988, p. 122).

Implica salir a la escena donde se actúan los dramas. El mundo, América y con ella los países por los que nos entregamos a esta propuesta filosófica, son eso: escenarios $\mathrm{y}$ arena donde nos jugamos de alguna manera la misma vida. Venir al mundo, nacer a un movimiento, a un proyecto y salir al escenario para actuar el drama del "otro", y las prácticas necesarias, son casi lo mismo.

Este quinto momento es el más importante, en cuanto representa la madurez o a una lenta agonía. ¿Cuál de ellas, madurez o agonía, se ha conseguido a los 40 ? 
Las tareas que trae nacer no terminan al entrar en escena, porque seguir existiendo implica una "función mundo-creadora adicional, gracias a la cual nosotros nos ligamos unos con otros, en calidad de miembros de una comunidad: la de los portadores del peso del mundo. Defino esta situación como el a priori de la transmisión" (Sloterdijk, 1988, p. 143).

Sloterdijk habla de dos momentos posteriores: la transmisión o comunicación, y la absolución o promesa, que no desarrollaré aquí porque implicaría ir más allá de las dos décadas que abarca esta reflexión. Pero conviene decir que nacer se convierte en crecer, madurar, prometer y también volverse a equivocar.

Dos textos hacen parte de esa transmisión y de pronto también de la promesa. Aquí comparto apartes de cada uno que conservo en un archivo personal: el primero, dirigido a todos los participantes del III Congreso de Filosofía Latinoamericana en 1984, por el entonces presidente de la República de Colombia, Belisario Betancur Cuartas.

La reflexión que ustedes conducen, busca las raíces del ser latinoamericano, no solamente a través de la historia del pensamiento -desde las culturas precolombinas hasta el pluralismo del presente-, sino mediante una praxis que edifique una ética de la convivencia y la solidaridad.

Solamente lograremos un puesto en la historia, cuando descubramos las raíces de nuestro ser; cuando se nos revele nuestra autenticidad ontológica y cultural: pero esta revelación solo se producirá, a través del crisol de la reflexión universal, de la confrontación de lo nuestro con lo perenne.

Autenticidad y universalidad son las dos caras del ser, que se revela ya desde la perspectiva de lo histórico concreto ya desde la luz de lo intemporal (Betancur, 1984).

Desde entonces la dinámica hasta hoy discutida: de lo situado a lo plural y general y los esencialismos de la identidad.

El segundo texto, enviado al IV Congreso de la Autocrítica. Una hoja volante titulada Nueva Nación y firmada por la Comisión de Acción Política y Social Álvaro Fayad Delgado, remitido por el Movimiento 19 de Abril (M-19) entonces sin desmovilizar. 
Decía en sus apartes:

Coincidimos con ustedes, en la necesidad del diálogo fecundo lejos de dogmatismos que nos impidan pensarnos y crearnos, desde nosotros mismos, como latinoamericanos. El aporte, compatriotas, que desde diversas posiciones conceptuales y quizá de práctica política están ustedes haciendo al proceso histórico de cambio en nuestro país y en nuestra América, lo comprendemos en su exacta dimensión. Tengan la certeza de que cada niño, mujer y hombre del M-19 les acompañarán, con un clavel en la mano y una voluntad indoblegable de realización exitosa (M-19, 1986).

Uno de los autores de esta página, Víctor Hugo Ardila Ariza, escribió 26 años después un bello texto filosófico, relacionado con uno de los temas de este congreso:

La filosofía es una mujer preñada del instante, El instante es la medida del amor. Como la esperanza, la filosofía es una mujer saturada de porvenir. Por ello (al igual que sucede con el primer amor: siempre estará adelante), el pensamiento filosófico es no solo libre continuación sino anticipación del futuro (Ardila, 2012, p. 28).

Para finalizar, hacemos una invitación a todos, a los que aún resistimos, a los que persistimos, a los que asisten, para que hagamos posible que haber venido al mundo de la filosofía no haya sido en vano, sobre todo para otros, para tantos que dependen de nuestra resistencia y que ese venir al mundo signifique también venir a la palabra que obra justicia, reconocimiento.

Escuchemos las voces jóvenes que hablan por ejemplo de superar escollos, los excesos de la institucionalización, de la profesionalización que nos vuelve más técnicos y especialistas que maestros, que contagian con las ganas de pensar, de imaginar, más que de repetir. Voces, que han denunciado situaciones como: vacío de tradición, marginamiento de lo real social, turismo intelectual por las edades del pensamiento: medievalistas, modernistas; monadismo pensante de autores, de programas, de facultades. Diagnóstico de Damián Pachón Soto (2011).

Fieles a las intencionalidades del comienzo: liberación y América con todos los pueblos hermanados en una realidad social necesitada, hagámonos capaces de insertarnos en 
un verdadero orden político donde se cruzan tantos campos materiales, de modo que las opciones que haga cada uno, cada grupo, institución, país, continente, escuche y responda las necesidades de cada comunidad y ethos cultural.

Como escribe el maestro Enrique Dussel (2009) en su Arquitectónica, vol. II:

Será necesario "ascender de lo abstracto" (la Arquitectónica) “a lo concreto" (la Crítica), donde todo ganará en complejidad, en contradicciones, en conflictos, en transformaciones necesarias institucionales, y hasta liberadoras en algunos casos. Todo esto nos exigirá un nuevo tipo de desarrollos categoriales de una innovada filosofía política crítica (Dussel, 2009, p. 523).

La tarea no ha terminado. Este es el relato de una manera de ver el comienzo, cuando con las deficiencias y las fallas reconocidas y atribuidas, pasamos por la experiencia de un parto o desligamiento que se hizo urgencia, iniciativa, luego pausa, autocrítica, posposición y finalmente arena de la lucha; pero quiere seguir siendo comunicación y compromiso.

Hagámoslo mancomunadamente con todos los que trabajan en temáticas y prácticas de género, de estudios culturales, de complejos proyectos decoloniales, en un continente y un mundo donde la vida se degrada, se obliga a abortar, se fusila, se decapita, se bombardea, pero donde seguimos buscando hacer efectivo lo que nos enseñara Kusch (1978):

Y es que en el fondo (de América) se tropieza violentamente con el milagro de ser hombre, con su profunda e inalienable vejez de serlo, pero que hace recién a partir de aquí a un futuro denso, auténtico, fundado, aunque nunca se sepa cuál es el fundamento. Se trata en el fondo de abrebar (sic) en nuestro estar lo fundado. Pero para ello nada se necesita. Apenas asumir toda la paradoja del vivir mismo, pero también toda su sacralidad (p. 146).

Recordando a Don Juan de Castellanos (como se citó en Ospina, 1999) "su gracia nos de Dios y sus favores // para llorar el tiempo malgastado" (p. 382). Porque esta América es: "tierra de bendición clara y serena // tierra que pone fin a nuestra pena" (p. 118). 
Y lo poco o mucho que hayamos hecho, como cantó Borges de las cosas comunes: "durarán más allá de nuestro olvido // no sabrán nunca que nos hemos ido" (p. 187).

\section{Referencias}

Ardila, H. (2012). De filosofía .Una mujer grávida del instante. Bogotá: Giordano Bruno editores, Arfo.

Assman, H. (1981, junio). Análisis de las necesidades básicas del hombre. En I Congreso Internacional de Filosofía Latinoamericana, junio 15-21 de 1980. Bogotá, Colombia: Universidad Santo Tomás.

Castro-Gómez, S. (2011). Crítica de la razón latinoamericana. Bogotá: Editorial Pontificia Universidad Javeriana Pensar.

Dussel, E. (2009). Política de la liberación. Arquitectónica, 2. Madrid: Trotta.

Dussel, E. (1983, enero-marzo). Carta al director de Cuadernos de Filosofía Latinoamericana, (14), 97-100. Bogotá: Ediciones USTA.

Foucault, M. (1974). La arqueología del saber. México: Siglo XXI.

Houghton, T. (2005). Cuerpo y palabra. Cuadernos de Filosofía Latinoamericana, 26, 27-36.

Kusch, R. (1978). Esbozo de una antropología filosófica americana. Buenos Aires: Ediciones Castañeda.

Marquinez, G. (1993). La Filosofía en América Latina. Bogotá: Editorial El Búho.

Marquínez, G. (2015, junio). ¿Qué es eso de Filosofía Latinoamericana? El Grupo de Bogotá. Conferencia en el Seminario de Investigación Xavier Zubiri.

Mignolo, W. (2009, enero-agosto). La idea de América Latina (la derecha, la izquierda, y la opción decolonial). Crítica y Emancipación, (2), 251-276. Recuperado de http:// bibliotecavirtual.clacso.org.ar/ar/libros/secret/CyE/CyE2/09idea.pdf

Movimiento 19 de abril (1986, julio). Nueva Nación. Documento inédito.

Ospina, W. (1999). Las auroras de sangre. Bogotá: Editorial Norma. 
Pachón, D. (2011). Balance y perspectivas de la filosofía en Colombia. En Estudios sobre el pensamiento colombiano, 1. Bogotá: Ediciones desde abajo.

Palacio, M. (2005). Vida y mundo: reflexión a partir de Dilthey y Husserl. Cuadernos de Filosofía Latinoamericana, 139-153. . (1980, junio). Ponencias. I Congreso Internacional de Filosofía Latinoamericana. Bogotá, Colombia: Universidad Santo Tomás.

Salazar, R. (1981). Presentación libro de Actas del I Congreso Internacional de Filosofía Latinoamericana, junio 15-21 de 1980. Bogotá: Ediciones USTA.

Salazar, R. (1983, enero-marzo). Acerca de la Filosofía Latinoamericana en la última década en Colombia. En Revista Cuadernos de Filosofía Latinoamericana. (14). Bogotá: Ediciones USTA.

Salazar, R. J. (1986, julio). Escritos sobre fenomenología. En Cuadernos de Filosofía Latinoamericana, (28-29), 206-208. Bogotá: Ediciones USTA.

Sloterdijk, P. (2006). Venir al mundo, venir al lenguaje. Lecciones de Frankfurt. Valencia: Pre-Textos.

Urbina, F. (1992). Las hojas del poder. Bogotá: Universidad Nacional.

Zabalza, J. (1981, junio). En Actas del I Congreso de Filosofía Latinoamericana, junio 15-21 de 1980. Bogotá: Ediciones USTA.

Zabalza, J. (1993). Filosofía de la historia e historia de las ideas. En La Filosofía en América Latina. Bogotá: Editorial El Búho.

Zabalza, J. (1986, enero-marzo). La normalización de la filosofía en Colombia y la bibliografía filosófica colombiana del siglo XX. En Cuadernos de Filosofía Latinoamericana, (26), 9-14. Bogotá: Ediciones USTA. 